\title{
Don't forget the jumper's knee in the young sportsman: evaluation of patellar tendinopathy with a high frequency ultrasound probe
}

\author{
B. Ruaro', ${ }^{1,2}$ M. Cutolo ${ }^{1}$, E. Alessandri', F. Zaottini ${ }^{3}$, R. Picasso ${ }^{3}$, F. Pistoia ${ }^{3}$, \\ G. Ferrari', C. Martinoli ${ }^{3}$
}

${ }^{1}$ Research Laboratory and Academic Division of Clinical Rheumatology, Department of Internal Medicine (Di.M.I.), Polyclinic Hospital San Martino, University of Genova, Italy; ${ }^{2}$ Cardinal Ferrari Centre, S. Stefano Rehabilitation, Fontanellato (PR), Italy; ${ }^{3}$ Division of Radiology, Polyclinic Hospital San Martino, University of Genova, Italy

\section{SUMMARY}

Patellar tendinopathy, or Jumper's knee, is a painful knee condition caused by inflammation of the patella tendon. This condition is most frequently observed in subjects who play sports that require repetitive regular jumping. Jumper's knee is frequently misdiagnosed as a minor injury and many athletes, like our patient, keep on training and competing and either tend to ignore the injury or attempt to treat it themselves. However, jumper's knee is a serious condition that requires a correct and timely diagnosis, which often necessitates ultrasound investigation in order to start the most appropriate treatment.

Key words: Patellar tendinopathy; Jumper's knee; ultrasound evaluation; color Doppler signal.

Reumatismo, 2019; 71 (3): 160-162

\section{CASE REPORT}

30-year-old man consulted our rheumatology division in June 2018 for pain in both knees. The pain, which was unresponsive to analgesic drugs, had started in about 2010, after 24 months of professional volleyball playing. Although he had suspended his competitive activity, he decided to play amateur volleyball in 2015 , but was forced to stop again due to the reappearance of knee pain. The patient made several rheumatology consultations over an 8-year period. The presence of concomitant diarrhea and recurrent oral ulcers led to a preliminary diagnosis of arthritis, linked to an inflammatory bowel disease. Azathioprine was prescribed without beneficial effects and he suspended the drug himself after only 2 months.

Corresponding author:
A diagnosis of reactive arthritis was then made at another consultation, due to a positive urine culture for Ureaplasma parvum and oral therapy with doxycycline, prednisone and cyclosporine was started. How- ever, as this treatment was also ineffective, once again the patient stopped the therapy. In 2017, he consulted an orthopedic specialist complaining of shoulder pain and was treated by intraarticular infiltrations with hyaluronic acid, followed by physiotherapy. Although the pain intensified after practicing sports, it was sometimes present also at rest, especially in the morning and at night. The patient's clinical history was silent due to the presence of patella misalignment or Osgood-Schlatter Disease as also observed in his previous radiographs. At physical examination, both active and passive mobilization of the knees and digital-pressure of the lower pole of both patella was painful. There was no swelling or warmth on palpation and the clinical examination of the other areas was unremarkable. The patient weighed $73 \mathrm{~kg}$ and was $180 \mathrm{~cm}$ tall. Laboratory tests for inflammation indices, rheumatoid factor, antinuclear antibodies and serological markers for celiac disease were within the normal range, as in the past. Longitudinal and transverse grey 
scale scans of the anterior aspect of the knees were performed using a linear 18-4 $\mathrm{MHz}$ transducer (Philips Healthcare Epiq 7). The US evaluation evidenced swollen, inhomogeneous and hypoechoic patellar tendons at the level of the insertion on the rotula. These changes were more evident in the left knee (Figure 1A and B, right and left knee, respectively). Color Doppler evaluation evidenced marked signs of neovascularization (Figure $2 \mathrm{~A}$ and $\mathrm{B}$, right and left knee, respectively).

On the basis of the patient's history, clinical examination and US, a diagnosis of patellar tendinopathy (i.e. Jumper's knee) in a subacute-chronic phase was made as the US evidenced an increased Doppler flow associated with tendinopathy (1-4).

At time of writing, our patient has been on pharmacological treatment with naproxen for some days to reduce the inflammation and pain at the tendon level, in line with literature and in agreement with the physiatrist, physio-kinesiotherapy, combined laser and Tecar therapy with some isometric exercises have been started (5-8). All his other physical-activities have been suspended.

The patient's condition has progressively improved and is, to date, stable. The pharmacological treatment has reduced his symptoms and the patient's clinical schedule allowed for a re-evaluation after two weeks to decide how to continue. As reported by several authors, the next rehabilitation steps will include exercises to gradually improve strength, as well as the power and speed of muscle contraction (7-9). The patient has also been advised to do sportspecific training and to learn how to manage his symptoms to prevent flare (7-9).

\section{DISCUSSION AND CONCLUSIONS}

Several studies have reported various nontraumatic underlying causes of anterior knee pain (10-12). In our personal experience, in agreement with the literature, they include rheumatoid arthritis, reactive arthritis, psoriatic arthritis, quadriceps tendinitis, patella chondromalacia and patellar subluxation/ dislocation, Osgood-Schlatter disease, Sind-

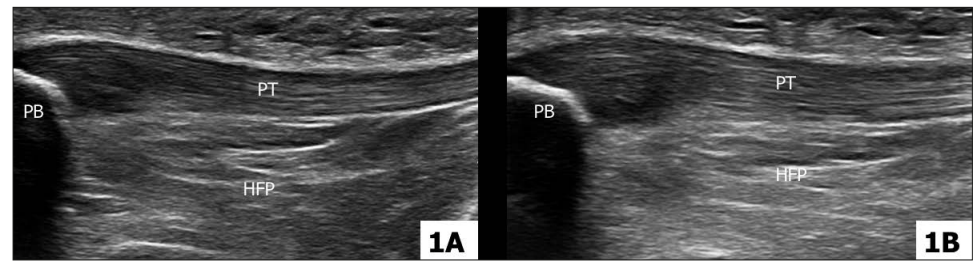

Figure 1 - $A$ and $B$ ) Longitudinal image of the anterior aspect of the knee showing the patellar tendon and the patellar profile. The insertional portion of the tendons is swollen and hypoechoic (PT, patellar tendon; PB, patellar bone; HFP, hoffa fat pad).

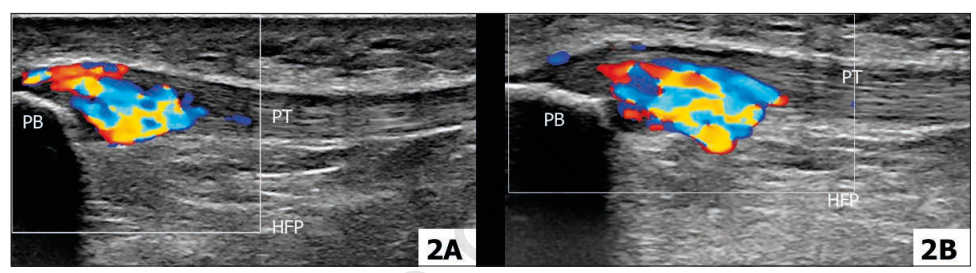

Figure 2 - A and B) Color Doppler detects signs of neovascularization in the affected area.

ing-Larsen-Johansson syndrome and patellar tendinopathy (i.e. Jumper's knee) (1012). Although both the history and physical examination are pivotal to excluding other causes of knee pain, ultrasound facilitates the differential diagnosis and whenever the US is unable to help in establishing the correct lesion site, the use of a second level investigation, such as magnetic resonance imaging (MRI), is recommended $(2,4,12)$. An ultrasound evaluation, characterized by hypertrophied and hypervascular synovium in the articular knee recesses or, less frequently, within the suprapatellar and infrapatellar bursa and bone erosions, suggest a diagnosis of rheumatoid arthritis. Enthesopathy is a distinctive feature of spondyloarthropathies (2-4).

Sonographic evaluation with high-resolution transducers has evidenced enthesopathy as having a heterogeneous, hypoechogenic texture, with thickening of the entheses, intratendinous calcific deposits and an alteration of the bone profile $(2-4,13)$. US is able to identify the involved area in the presence of quadriceps tendinitis, whilst X-rays, MRI and arthroscopy are the best methods to identify chondromalacia of the patella, patellar subluxation or dislocation and Osgood Schlatter Disease and/or Sinding-LarsenJohansson syndrome $(10,11,14)$. 
As reported in our case, the ultrasound examination is a reliable diagnostic tool for patellar tendinopathy, as it is very sensitive in detecting subtle changes in the presence of a superficial fibrillary tendon, parallel to the skin $(7,10,11,14)$.

The sonographic findings differ according to the tendinopathy phase. In fact, although the tendon is thickened with a normal echo structure in acute tendinosis, the fibrillary pattern is lost with disease progression and the tendon becomes hypoechoic, with further swelling (2-4, 14). Increased Doppler flow can be an indication of hyperemia that may be associated with tendinopathy, especially in the subacute-chronic phase, when the intratendinous neovascularization starts. Some authors have reported that the process of neovascularization is associated with the onset of painful episodes $(2-4,14)$.

The primary aim of reporting our case is that of emphasizing the importance of taking patellar tendinopathy into consideration when making a differential diagnosis in the presence of bilateral knee pain. It also aims to emphasize that jumper's knee should not be underestimated as a minor injury since it is a serious condition requiring timely attention to avoid irreparable damage and/or the need for surgical repair. Although patellar tendinopathy (Jumper's knee) is a frequent, disabling condition affecting mostly athletes, it is also frequently observed in the non-athletic population $(10,11,14,15)$.

Moreover, this case shows how ultrasound examination of the muscle and tendon can help make a correct clinical diagnosis, as it can identify the structures involved in the pathological process should the physical exam be inconclusive.

Acknowledgements: The authors would like to thank Barbara Wade, contract Professor at the University of Torino, for her linguistic advice. Barbara Ruaro was supported by a Grant from the European Society of Rheumatology.

\section{Conflicts of interest: None.}

\section{REFERENCES}

1. Lian O, Engebretsen L, Ovrebø RV, Bahr R. Characteristics of the leg extensors in male volleyball players with jumper's knee. Am J Sports Med. 1996; 24: 380-5.

2. Gemignani M, Busoni F, Tonerini M, Scaglione M. The patellar tendinopathy in athletes: a sonographic grading correlated to prognosis and therapy. Emerg Radiol. 2008; 15: 399404.

3. Martinoli C, Bianchi S, Dahmane M, et al. Ultrasound of tendons and nerves. Eur Radiol. 2002; 12: 44-55.

4. Khan KM, Bonar F, Desmond PM, et al. Patellar tendinosis (jumper's knee): findings at histopathologic examination, US, and MR imaging. Victorian Institute of Sport Tendon Study Group. Radiology. 1996; 200: 821.

5. Rodriguez-Merchan EC. The treatment of patellar tendinopathy. J Orthop Traumatol. 2013; 14: $77-81$.

6. Everhart JS, Cole D, Sojka JH, et al. Treatment options for patellar tendinopathy: a systematic review. Arthroscopy. 2017; 33: 861-72.

7. Rudavsky A, Cook J. Physiotherapy management of patellar tendinopathy (jumper's knee). J Physiother. 2014; 60: 122-9.

8. Kettunen JA, Kvist M, Alanen E, Kujala UM. Long-term prognosis for jumper's knee in male athletes. A prospective follow-up study. Am J Sports Med. 2002; 30: 689-92.

9. Peters JA, Zwerver J, Diercks RL, et al. Preventive interventions for tendinopathy: a systematic review. J Sci Med Sport. 2016; 19: 205-11.

10. Ferretti A. Epidemiology of jumper's knee. Sports Med. 1986; 3: 289-95.

11. Almekinders LC, Temple JD. Etiology, diagnosis, and treatment of tendonitis: an analysis of the literature. Med Sci Sports Exerc. 1998; 30: 1183-90.

12. Calmbach WL, Hutchens M. Evaluation of patients presenting with knee pain: Part II. Differential diagnosis. Am Fam Physician. 2003; 68: 917-22.

13. van der Worp H, van Ark M, Roerink S, Pepping GJ, van den Akker-Scheek I, Zwerver J. Risk factors for patellar tendinopathy: a systematic review of the literature. Br J Sports Med. 2011; 45: 446-52.

14. Fredberg U, Bolvig L. Jumper's knee. Review of the literature. Scand J Med Sci Sports. 1999; 9: 66-73.

15. Lian OB, Engebretsen L, Bahr R. Prevalence of jumper's knee among elite athletes from different sports: a cross-sectional study. Am J Sports Med. 2005; 33: 561-7. 